\title{
Programming experiments with pocket programmable calculators
}

\author{
GEORGE H. ROBINSON \\ University of North Alabama, Florence, Alabama 35630
}

\begin{abstract}
The use of programmable calculators as research equipment for stimulus presentation and response recording, in addition to their use in mathematical and statistical analysis, is proposed. The calculators may be used alone or in conjunction with other equipment in programming experiments.
\end{abstract}

Pocket programmable calculators are used widely in conventional mathematical and statistical analysis. Their potential use is not, however, limited to computation. They can also be used as programming and stimulusgenerating apparatus to arrange experimental conditions in predetermined sequences, to present visual (numerical) stimuli, to record, test, and accumulate subject-entered responses, and to decide future trial conditions contingent upon previous trials and responses. Illustrating these capabilities, programs have been prepared and tested for the Texas Instruments SR-56 and TI-58 programmable calculators for several experimental tasks.'

\section{EXPERIMENTAL TASKS USING THE CALCULATOR}

\section{Short-Term Memory for N-Digit Numbers}

This is essentially the Peterson and Peterson (1959) procedure, but with numbers (which, for a trial block, may vary in length from 3 to 10 digits) to be recalled instead of letters. The distractor task consists of having the subject vocally square each of a series of regularly presented random digits until the expiration of the recall interval, at which time the two-digit number " 11 " is displayed to signal the subject to key in the number recalled. Pressing the R/S key tests for correctness, adds one to the appropriate accumulating register, and initiates the next trial (or stops following the last trial).

\section{Two-Interval Forced-Choice Time Discrimination}

In this task (which is similar to the procedure of Penner, 1976, but for longer intervals), the subject indicates the longer of two blank intervals, each of which begins and ends with either a " 4 " (for the first interval) or a " 5 " (for the second interval) in the display. Responses of " 4 " or " 5 " followed by $\mathrm{R} / \mathrm{S}$ are tested and accumulated, the trial number is displayed, and the next trial is initiated. The interval between the second " 4 " and the first " 5 " is independently adjustable for possible short-term memory investigations.

\section{Single-Interval Time Discrimination}

An interval of time is demarcated by a display of " 8888 " at the start and end of the interval, and the subject decides whether it is of a specified length or longer (based on Kinchla, 1972). The response of " 0 " and $\mathrm{R} / \mathrm{S}$ (if it is believed to be the specified length) or "I" and R/S (if longer) is followed by (optional) feedback, trial number, and the randomly selected stimulus interval of the next trial.

\section{Digit Span Memory}

Random numbers varying randomly in length from six to eight digits are presented from trial to trial for a predetermined number of trials. The display duration in a block of trials can be $.5,1$, or $1.5 \mathrm{sec}$. The subject keys in the number remembered and presses $\mathrm{R} / \mathrm{S}$, which initiates the next trial.

\section{Extrasensory Perception}

The task is to enter the number from the set of digits, $1-5$, that matches a randomly selected number from that set stored within the calculator. Feedback following responses is optional.

For each of the representative programs, separate registers continuously accumulate numbers of correct and incorrect responses. These data are also separately accumulated for each digit length in the digit span memory program. Each program was originally developed for the SR-56 and, thus, consists of 100 or fewer program steps and uses 10 or fewer data memories, in accordance with the limits of the SR-56. The TI-58 and TI.59 calculators, among other, more recent calculators, have many more program steps and data memories, thereby permitting more complex experimental tasks to be programmed.

\section{EXPERIMENTAL TASKS USING THE CALCULATOR WITH AN OPTICAL INTERFACE}

Thus far the discussion has dealt with the use of the calculator alone for presenting stimuli and recording responses. By coupling the calculator output with conventional research equipment, the versatility of programming can be combined with the diversity of stimuli used in behavioral research. Photocells can be effectively used to accomplish this without altering the calculator. 


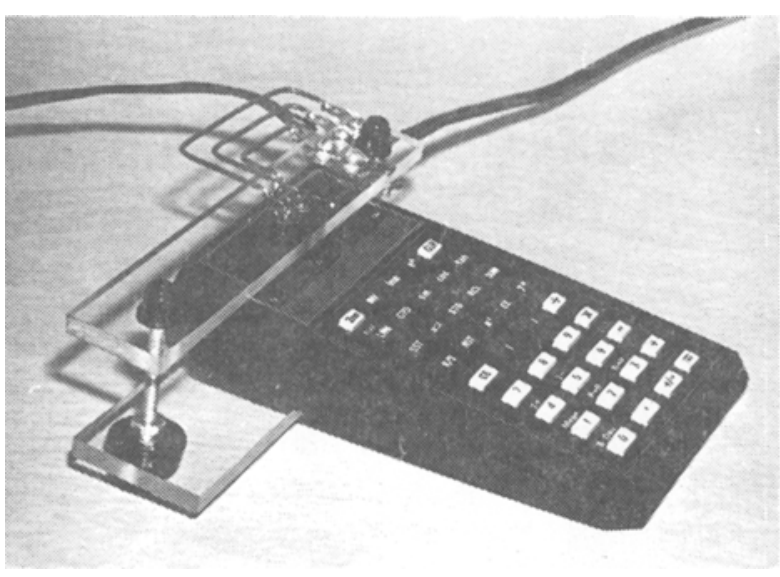

Figure 1. Prototypical optical interface with two photocells installed and positioned over the calculator display.

Figure 1 shows an optical interface consisting of two Clairex CL904L photocells mounted in a $1 / 4$-in. Plexiglas plate fastened in position over the SR-56 display. Placement is such that the photocells are directly over two nonadjacent digits in the calculator display. The CL904L photocell was selected on the basis of its size, speed of response, and spectral sensitivity (corresponding to the calculator's LED spectral output). Extraneous light from unused digits of the calculator display is controlled by painting the internal surface of the mounting hole for each photocell with India ink; ambient illumination is controlled by covering the optical interface with a shield (e.g., a dark cloth).

\section{Classical Conditioning}

Using the optical interface with a dual photocell amplifier driving stimulus sources, programs for the SR-56 and TI-58/59 have been developed for classical conditioning (delayed, trace, or backward). The programs (1) generate a variable intertrial interval (ITI), (2) trigger CS presentation, (3) generate the interstimulus interval between CS and US onsets (ISI), (4) trigger the presentation of the US, and (5) repeat all of this for a predetermined number of trials and then stop. A simple alteration permits presentation of a specific number of extinction trials or US sensitization control trials. The amount of desired ITI variability (as a control for temporal conditioning) can be adjusted from $0 \%$ to nearly $100 \%$ of the mean ITI.

\section{PROGRAMMING CONSIDERATIONS AND DISCUSSION}

Timing in these programs is, in most cases, accomplished with decrement-and-skip-on-zero loops (dsz loops). Accuracy determinations have been made for the SR-56 and TI-58 calculators using the optical interface, an amplifier, and a frequency counter. Ten trials were conducted with each of $1,2,5,10,20,50,100,200$, 500 , and 1,000 dsz loops. The maximum percentage error (defined here as three times the coefficient of variation, i.e., $300 \mathrm{~S} / \mathrm{M}$ ) observed was $.268 \%$ for the SR-56 and $.021 \%$ for the TI-58. ${ }^{2}$

By trying various numbers of dsz loops and measuring the consequent time intervals generated, one can derive a least-squares linear equation that can be used to estimate the number of dsz loops required for a desired interval. For example, if $t$ represents ITI in seconds in the classical conditioning program (with constant ITI) and $\mathrm{k}$ is the number of dsz loops, the equation relating these (for the SR-56 used) is $\mathrm{k}=11.45 \mathrm{t}-39.38$. Thus, for a 60 -sec ITI 648 loops are required. Verification is done with a stopwatch, with subsequent modification, if necessary, of the number of loops needed.

Randomization is accomplished through pseudorandom number algorithms. An example used in these programs is to take the first $\mathrm{N}$ digits of the fractional part of $[s+(i / \pi)]^{2}$, where $s$ is a seed number (e.g., any number from -5 to +5 ) and $i$ is the ordinal number of the pseudorandom number generated. ${ }^{3}$ The preprogrammed random number generating capability present in the TI-58/59 calculators can be accessed as a subroutine with a consequent reduction of program steps.

Desired features of calculators to be used in programming experiments include dsz looping, conditional and unconditional branching, pause, blanked display when running a program, LED display (if an optical interface is planned), integer/fraction, and as many data memories and program steps as possible. The main limitation of the method described here is the restriction on the precision of timed intervals. Each dsz loop takes about $90 \mathrm{msec}$ in the SR-56 and $170 \mathrm{msec}$ in the TI-58; more precise timing is possible by adding other "do-nothing" functions within or outside the loop (e.g., the NOP function consumes $16 \mathrm{msec}$ in the SR-56 and TI-58, others longer).

The programming capabilities described are intended to be illustrative and not exhaustive. Numerous other experiments, as well as variations of those described, can be programmed similarly. Even for experiments having more stringent timing requirements, the pocket programmable calculator can frequently be used in preliminary work to determine experimental parameters (pilot studies) without tying up more expensive apparatus. Conventional programming equipment, larger desk programmable calculators, or microcomputers (Bloch, 1978) could subsequently be used in the actual experiment, with adequate timing thereby provided.

\section{REFERENCES}

BLock, R. M. Aspects of programmable calculator stimulus control and data acquisition in a psychophysiology laboratory. Behavior Research Methods \& Instrumentation, 1978, 10, 246-248.

KinchlA, J. Duration discrimination of acoustically defined intervals in the 1 - to $8-\mathrm{sec}$ range. Perception \& Psychophysics, $1972,12,318-320$. 
Nievergelt, J., Farrar, J. C., \& Reingold, E. M. Computer approaches to mathematical problems. Englewood Cliffs, N.J: Prentice-Hall, 1974.

Penner, M. J. The effect of marker variability on the discrimination of temporal intervals. Perception \& Psychophysics, 1976, 19, 466-469.

Peterson, L. R., \& Peterson, M. J. Short-term retention of individual verbal items. Joumal of Experimental Psychology, 1959, 58, 193-198.

\section{NOTES}

1. Copies of the programs mentioned are available from the author. The programs for the TI-58 can be used without modification on the TI-59 and with appropriate modifications on other calculators using algebraic logic. Calculators using reverse Polish notation (RPN), such as those of the Hewlett-Packard family, will require translation of the programs to the RPN logic. The failure of the Hewlett-Packard calculator displays to blank out when running a program limits their usefulness for tasks described in this article.
2. By way of comparison, the percentage error for a crystalclock-driven LED with an output adjusted to be equal in effect on the photocell to that of the calculator display was $.004 \%$.

3. This algorithm consumes fewer program steps than a more conventional algorithm (see Nievergelt, Farrar, \& Reingold, 1974, Chapter 4, for a discussion of random number generation). An analysis of 1,000 sets of 100 one-digit numbers produced with this algorithm, with values of $s$ from .01 to 10.00 in .01-increments, revealed 23 of the $1,000 x^{2}$ values to be significantly nonuniform at the .050 level of significance, as opposed to 50 expected by chance. It appears, therefore, to meet the uniformity criterion. The number of random numbers available by means of this algorithm depends upon the calculating capacity of the machine, the number of digits in each random number, and the seed number. For seed numbers with absolute values less than 1.0, the SR-56 and TI-58 will generate over 3 million one-digit, but only approximately 300 ninedigit, numbers.

(Received for publication September 27, 1978; revision accepted December 28,1978 .) 